\title{
Tetraplegia secondary to lumbar epidural punture: A case report
}

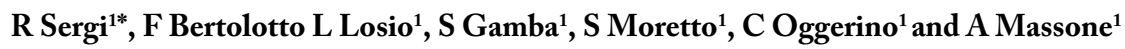 \\ Department of Rehabilitation, Spinal Cord Injury Unit, Pietra Ligure, Savona, Italy
}

\begin{abstract}
Background: To illustrate a rare complication connected to lumbar puncture practice that has involved the cervical spinal cord.

Methods: We illustrate the case of a 60 years old male, who has been submitted to lumbar puncture in order to treat lumbar sciatica, and who has presented, a few hours after the treatment, the onset of quadriplegia.

Results: Analysis of the cerebrospinal fluid, of the imaging and the data from the laboratory have excluded an infective-inflammatory pathology, autoimmune or from a demyelinating disease. Image diagnostics has shown, as well as an evident medullar lesion that the patient was the bearer of an unknown stenosis of the cervical canal.

Conclusion: The documented stenosis of the cervical canal, as far as we are concerned, could be a pre-existing cause of the setting up of the clinical picture. The etiopathogenetic hypothesis that we are putting to the judgment of the readers, in our opinion the most likely, is that the medicine, inserted by mistake into the cerebrospinal cavity, has been conveyed upward by the normal cerebrospinal flux, and has concentrated at the point of the cervical stenosis, where it has exerted its
\end{abstract} toxic effects on the medulla.

\section{Introduction}

Spinal anaesthesia seldom causes damages to the spinal cord [1-4]. When it happens the causes can be schematized as follows:

1) Direct medullar trauma caused by the needle, the catheter or by the toxic effects of the local anaesthetic

2) Spinal hematoma, particularly in presence of coagulating disorders, vascular malformations, tumour

3) Medullar ischemia

4) Infections: epidural abscess, bacterial meningitis

Our objective is to illustrate a rare complication connected to lumbar puncture practice that has involved the cervical spinal cord.

\section{Case report}

60 years old male, who worked as a bricklayer, On November $27^{\text {th }}$ 2007 the patient, who has been suffering of lumbar sciatica for months is submitted to lumbar puncture. The clinical report at our hands relates the puncture contained steroid and local anaesthetic, but it does not specify the molecules used. After the treatment, the patient goes back home driving his car. A few hours after the injection some neurological signs and symptoms like paresthesias and a worsening strength deficiency of the lower limbs, together with similar complaints of the wright upper limb justify the patient return and admission to the hospital. The Nuclear Magnetic Resonance performed the day after shows: stenosis of the rachidian canal with multiple focal obliteration both of the front and of the rear of the perimedullary fluid spaces and marks on the spinal cord. The last one appears diffusely altered from the bulb-medullary junction to the level of vertebra $\mathrm{C} 5$ (this is the point of maximum rachidian stenosis).

\section{Encephalon Magnetic Resonance: negative}

Sample of the cerebrospinal fluid: negative

There is no history of coagulating illness and of anterior neurological deficiency.

Admitted to our centre for rehabilitation on December $20^{\text {th }} 2007$ with neurological level right C5, left C6, A.S.I.A. (American Spinal Cord Association) impairment scale "B"

The patient did not show respiratory difficulty, but neurogenic bladder and bowels.

While hospitalized, the patient has completed the rehabilitation program dedicated to reach the largest autonomy and whereas possible to strengthen the muscular system.

On January $16^{\text {th }} 2008$ another Magnetic Resonance has been carried out in order to evaluate the development of the picture (Figure 1).

The imaging of the thoracic-lumbar sectors has been added to the evaluation of the cervical sector in order to reveal any possible concomitant lesions, but the result was negative. The cervical lesion appeared unchanged.

Follow up: neurological level of March $4^{\text {th }} 2008$ right C5, left C6, A.S.I.A. impairment scale " $\mathrm{C}$ ".

Correspondence to: Roberto Sergi, Spinal Cord Unit, Santa Corona Hospital Via XXV Aprile, 3817027 Pietra Ligure, SV, Italy, E-mail: r.sergi@asl2.liguria.it

Key words: lumbar puncture, tetraplegia

Received: August 22, 2017; Accepted: September 15, 2017; Published: September 18,2017 


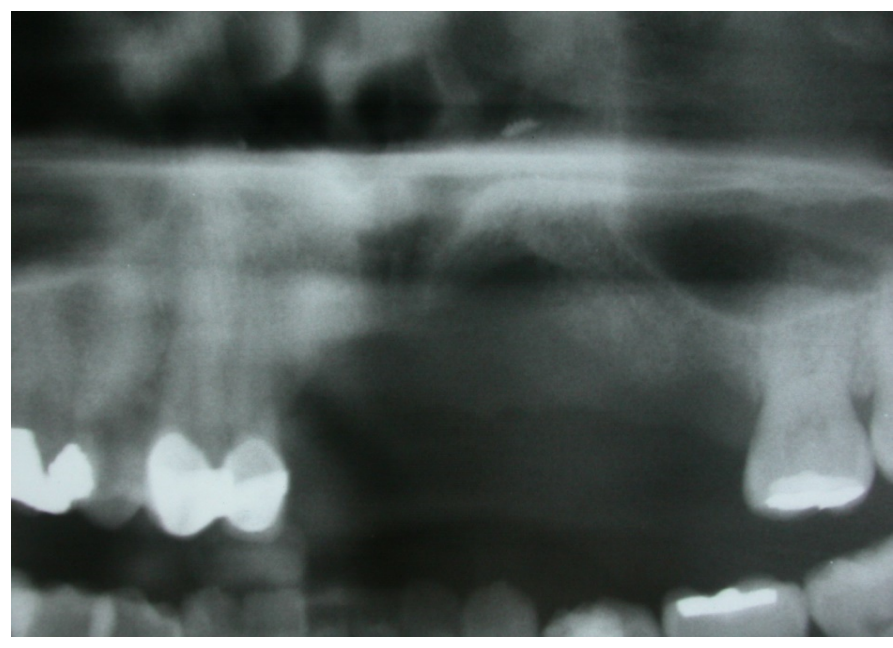

Figure 1. Magnetic resonance of lumbar epidural puncture

\section{Discussion and conclusions}

As far as we are concerned this case is important because for the first time a cervical medullary lesion consequent on a lumbar puncture is described. In the medical literature the association between lumbar punctures and medullary lesions, even though rare, are topographically related: a lumbar puncture generates a thoracic lumbar lesion or a cervical puncture generates a cervical lesion. The etiopathogenetic hypothesis that we are putting to the judgment of the readers, in our opinion the most likely, is that the medicine, inserted by mistake into the cerebrospinal cavity, has been conveyed upward by the normal cerebrospinal flux, and has concentrated at the point of the cervical stenosis, where it has exerted its toxic effects on the medulla. Should this case be confirmed by other studies, the presence of a stenosis of the canal should induce to practice lumbar puncture with particular attention, and the patient should be informed.

\section{References}

1. Cheney FW, Domino KB, Capian RA, Posner K (1999) Nerve injury associated with anesthesia. A closed claims analysis. Anesthesiology 90: 1062-1069. [Crossref]

2. Aromaa U, Lahdensuu M, Coazanitis DA (1997) Severe complications associated with epidural and spinal anaesthesias in Finland 1987-1993. A study based on patient insurance claims. Acta Anaesthesiol Scand 41: 445-52. [Crossref]

3. Flandin-Blety C, Barrier G (1995) Accident following extradural analgesia in children The results of a retrospective study. Paediatr Anaesth 5: 41-6. [Crossref]

4. Mapstone TB, Rekate HL, Shurin SB (1983) Quadriplegia secondary to hematoma after lateral C-1, C-2 puncture in a leukemic child. Neurosurgery 12: 230-1. [Crossref]

Copyright: $@ 2017$ Sergi R. This is an open-access article distributed under the terms of the Creative Commons Attribution License, which permits unrestricted use, distribution, and reproduction in any medium, provided the original author and source are credited. 